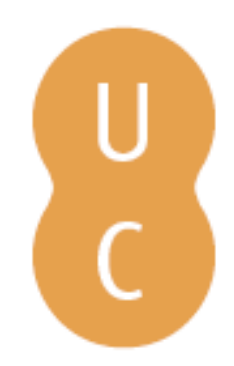

\title{
pommalina
}

\section{A Economia política da desinstitucionalização psiquiátrica}

\author{
Autor(es): Madianos, Michael \\ Publicado por: Imprensa da Universidade de Coimbra \\ URL \\ persistente: \\ URI:http://hdl.handle.net/10316.2/35955 \\ DOI: \\ DOI:http://dx.doi.org/10.14195/978-989-26-0852-5_2
}

Accessed : $\quad$ 26-Apr-2023 13:23:59

A navegação consulta e descarregamento dos títulos inseridos nas Bibliotecas Digitais UC Digitalis, UC Pombalina e UC Impactum, pressupõem a aceitação plena e sem reservas dos Termos e Condições de Uso destas Bibliotecas Digitais, disponíveis em https://digitalis.uc.pt/pt-pt/termos.

Conforme exposto nos referidos Termos e Condições de Uso, o descarregamento de títulos de acesso restrito requer uma licença válida de autorização devendo o utilizador aceder ao(s) documento(s) a partir de um endereço de IP da instituição detentora da supramencionada licença.

Ao utilizador é apenas permitido o descarregamento para uso pessoal, pelo que o emprego do(s) título(s) descarregado(s) para outro fim, designadamente comercial, carece de autorização do respetivo autor ou editor da obra.

Na medida em que todas as obras da UC Digitalis se encontram protegidas pelo Código do Direito de Autor e Direitos Conexos e demais legislação aplicável, toda a cópia, parcial ou total, deste documento, nos casos em que é legalmente admitida, deverá conter ou fazer-se acompanhar por este aviso. 

Michael Madianos 1

\section{A ECONOMIA POLÍTICA}

\section{DA DESINSTITUCIONALIZAÇÃO PSIQUIÁTRICA}

A desinstitucionalização dos Doentes Mentais Crónicos iniciou-se, em muitos países ocidentais, nos últimos anos da década de 60 e princípios da década de 70 do último século, claramente depois da introdução massiva dos neurolépticos.

Contudo, em muitos países, a saída dos doentes internados fez-se antes que a comunidade e a rede de apoio local tivessem organizado um número satisfatório de alternativas para alojar os pacientes que receberam alta, resultando no fenómeno negativo de "porta-giratória" e levando-os a situações de sem-abrigo.

Por outro lado, a desinstitucionalização está directamente ligada ao Estado Social e ao apoio financeiro adequado do programa. Em vários países, a transição de um Estado Providência para uma Economia de Mercado teve um impacto negativo acentuado na prestação de cuidados adequados de saúde mental aos indivíduos de classe socioeconómica baixa e vulnerável.

A desinstitucionalização comprovou ser bem-sucedida quando houve uma forte motivação ideológica e humanitária, quando a reforma psiquiátrica foi considerada uma prioridade e foi completada com um sistema abrangente de alternativas baseadas na comunidade e no suficiente apoio social.

\footnotetext{
${ }^{1}$ MD, MPH, DrMed FEPA, Professor de Psiquiatria do Departamento de Saúde Mental e Ciências do Comportamento - School of Health Sciences, University of Athens. Presidente da World Association for Psychosocial Rehabilitation. E-mail: madianos@ nurs.uoa.gr
} 


\section{Introdução}

Ao falar sobre desinstitucionalização dos doentes mentais crónicos, a primeira coisa que vem à mente é a origem das grandes instituições psiquiátricas, os asilos, no século XVIII. O aparecimento dos Asilos foi o resultado do medo ancestral contra o comportamento inexplicável, estranho e ritualístico, com inesperados episódios de violência e outras acções inadequadas de algumas pessoas; fenómenos que, desde longa data, têm sido relacionados com possessões diabólicas de acordo com a tradição Judaico-Cristã (Rothman, 1971).

O tratamento dos doentes internados nestes asilos esteve muito longe de poder ser considerado como humano. As características comuns de "tratamento" consistiam em medidas restritivas, tais como o uso de correntes, camas de palha, encerramento em celas pequenas e sujas, alimentação fraca e desnutrição causada por alguns remédios empíricos tais como a ipecacuana, antimónio tartarisado, xarope de pinheiro, valeriana e outras tisanas ervanárias. Os castigos corporais, incluindo a flagelação, eram aplicados frequentemente. Era este o chamado estado de "psychiatry" com excepção de alguns casos de instituições de caridade a que os historiadores deram o nome de "Centros de Tratamento Moral", locais como o York Retreat na Inglaterra, o Bonifacio Hospital em Florença (Itália), os Hospitais de Bicetre e Salpetriere em Paris, e o Asilo Bloomingdales, em Nova York. A situação nos grandes asilos psiquiátricos manteve-se imutável, embora tivessem sido transformados na sua maioria, em meados do século XIX, em Hospitais Psiquiátricos em muitos países ocidentais (Mora, 1975).

Levou mais de cem anos, marcados por acontecimentos e alterações dramáticas com vários problemas sociais, económicos e políticos, incluindo duas guerras mundiais catastróficas, para se chegar à negação teórica e prática dos asilos. É de notar que nos anos 40, vários profissionais de saúde mental com ideias inovadoras começaram a aplicar nos seus hospitais uma política de "portas abertas" e introduziram ideias e métodos novos para a reabilitação dos pacientes institucionalizados, incluindo o conceito de comunidade terapêutica (Rothman, 1971). A era da Psiquiatria Social e Comunitária foi desenvolvida nos anos 60 e 70, em muitos países 
ocidentais (Ralph, 1983). Foi naturalmente a introdução da clorpromazina e outros neurolépticos que facilitou a abertura dos portões da nova era (Mechanic, 2007).

Nos E.U.A., a taxa de internamentos em Hospitais Psiquiátricos por 100.000 habitantes declinou de 400 por 100.000 registados em 1935 para $200 \mathrm{em} \mathrm{1970.} \mathrm{As} \mathrm{alternativas} \mathrm{a} \mathrm{uma} \mathrm{vida} \mathrm{institucionalizada} \mathrm{em} \mathrm{hospi-}$ tais psiquiátricos são agora baseadas na comunidade com uma enorme variedade de serviços, tais como Hospitais de Dia, Centros Comunitários de Saúde Mental, Centros de Reabilitação Profissional, Cooperativas e Empresas de Inserção, Emprego Apoiado, Casas Halfway, Clubes Sociais, Casas de Repouso e Pousadas (Mechanic, 2007).

$\mathrm{Na}$ década de 70, o movimento de desinstitucionalização cresceu nos Estados Unidos e em alguns países europeus como na Itália, Grã-Bretanha, Suécia, França, Espanha e outros (Bachrach, 1976 \& Madianos, 2002). Contudo, em muitos países, as saídas maciças de doentes mentais crónicos foram feitas antes da comunidade e das redes de apoio local terem organizado um número satisfatório de alternativas para hospedar os pacientes a quem era dada alta hospitalar (Mechanic \& Rochefort, 1990). Os efeitos colaterais da desinstitucionalização foram nefastos para os doentes: verificou-se o "abandono" de pessoas com doenças mentais graves e a massificação dos "sem-abrigo". O factor responsável por esta situação foi o orçamento insuficiente para o sector da saúde mental, principalmente nos E.U.A. e em alguns outros países industrializados (Saraceno \& Barbui, 1997; Wave \& Goldfingers, 1997).

Assim, a situação da economia, ligada a uma adequada política de segurança social por parte do Estado, desempenha o papel principal na efectiva desinstitucionalização e reabilitação de pessoas portadoras de doenças mentais crónicas na comunidade (Thornicroft \& Bebbington, 1989).

\section{Economia e doença mental}

Há muito tempo que se sabe que as condições sociais e económicas influenciam directamente os tratamentos psiquiátricos e os seus resultados. 
O trabalho de Harvey Brenner demonstrou que a admissão de doentes no Hospital Psiquiátrico, no Estado de Nova York, especialmente por esquizofrenia, aumentou durante os períodos de recessão económica, embora a despesa anual dos hospitais psiquiátricos estaduais não tivesse diminuído de forma considerável (Brenner, 1973).

Tudo parecia indicar que os fracos recursos económicos das famílias e dos sistemas de apoio, juntamente com o desemprego existente, influenciavam a evolução da doença, impedindo a recuperação e provocando recaídas e reinternamentos. Nos anos 30, Faris e Dunham, num estudo ecológico que realizaram em Chicago, demonstraram que as maiores taxas de prevalência de tratamentos para a "Demência Precoce" estavam concentrados nas áreas suburbanas em torno da "grande" Chicago: 7 casos por mil adultos nas áreas suburbanas em contraste com os 2,5 por mil em áreas de classe socioeconómica alta. Este estudo foi replicado em várias outras cidades do E.U.A. com os mesmos resultados (Faris \& Dunham, 1939).

Nos anos 50, o estudo clássico de Hollingshead e Redlich, em New Haven, mostrou que pacientes de classe socioeconómica baixa tinham mais probabilidades de serem admitidos nos Hospitais Psiquiátricos e eram neles mais depressa readmitidos (Hollingshead \& Redlich, 1958). Uma década mais tarde, nos anos 60, J. Myers e L. Bean descobriram, novamente em New Haven, que os pacientes das classes desfavorecidas tiveram um maior tempo de permanência nos hospitais e foram reinternados com maior frequência; além disso, verificaram que ao ser-lhes dada alta do hospital apenas tinham recurso a trabalhos precários e condições de vida caracterizadas pelo isolamento e marginalização devido ao estigma (Myers \& Bean, 1968).

Num outro estudo realizado por Astrachan et al. (1974) verificou-se que os pacientes de classe social mais baixa apresentaram mais sintomas psicóticos num período de 2-3 anos de acompanhamento, após a alta do hospital. Acresce que nas populações de classe baixa a prevalência de acontecimentos stressantes, frequentes ao longo da vida, é mais elevada e, consequentemente, o risco de stress suscitador de um episódio agudo de esquizofrenia é maior (Yanos et al, 2001). 
Alguns outros estudos apontaram efeitos prejudiciais na saúde mental relacionados com a economia, o desemprego, as dificuldades económicas da comunidade, a instabilidade e a ruptura social, bem como a criminalidade e a violência (Wave \& Goldfingers, 1997, Saraceno \& Barbui, 1997, Wilton, 2007).

\section{Desinstitucionalização, reabilitação psicossocial e globalização da economia}

A relação entre a economia e a efectiva reabilitação psicossocial necessária aos pacientes desinstitucionalizados já foi comprovada. Por exemplo, o trabalho é um objectivo básico da reabilitação, e obter um emprego (a tempo integral ou parcial) é sempre uma ambição desejável. A questão é como conseguir um emprego quando a economia está em recessão e as taxas de desemprego são elevadas. Na maioria dos Estados Ocidentais, a recessão económica é um fenómeno crónico. Isso pode causar dificuldades até mesmo para os programas de emprego protegido (Drew et al., 2001). Além disso, a recessão económica está sempre ligada com os cortes orçamentais dos programas de reabilitação psicossocial.

A globalização da economia tem trazido sérias mudanças económicas (economia de mercado), aumentando as taxas de desemprego (as grandes empresas mudam-se para países de mão-de-obra barata), a privatização das instituições sociais (menos Estado de Bem-Estar Social), alta mobilidade social, transformações na estrutura familiar, e a marginalização dos grupos socialmente desfavorecidos (por exemplo, os trabalhadores não qualificados, os grupos minoritários e pessoas com deficiência) (Morrow, 2004 \& Madianos, 2006). A marginalização-alienação dos grupos sociais economicamente desfavorecidos, incluindo os deficientes mentais, que se verifica nas regiões metropolitanas dos países ocidentais, aumenta o risco de desenvolvimento de um transtorno mental crónico de agravamento do já existente (Williams et al., 1973).

A crescente insegurança económica provoca uma sobrecarga emocional e económica considerável, especialmente nas famílias em que um membro 
sofre de uma doença mental grave. Estas famílias muitas vezes enfrentam dificuldades económicas profundas (Madianos et al., 2004; Schene et al., 1998). A pobreza tem um impacto significativamente negativo sobre o bem-estar psicológico, a auto-estima, a saúde (física e mental) e na qualidade de vida em geral. Baixos níveis de qualidade de vida nos seus vários domínios (relações sociais, actividades de lazer, alimentação, alojamento) são vivenciados por pessoas que sofrem de doenças mentais graves e seus familiares (Wilton, 2004).

\section{A pobreza e o desenvolvimento dos cuidados de saúde mental e serviços de reabilitação em todo o mundo.}

No novo milénio, a maioria dos países do mundo pertence à categoria de nível baixo ou médio-baixo no que respeita ao desenvolvimento económico. Essas condições económicas e sociais refletem-se actualmente nos cuidados de saúde mental e serviços de reabilitação psicossocial nesses países. Por outro lado, o aumento da incidência de transtornos mentais está a impor um grande fardo global (Murray \& Lopez, 1996).

Tabela 1. Distribuição dos países do mundo de acordo com seu nível económico e a existência de serviços psiquiátricos integrados na comunidade, prestações de invalidez e de serviços especializados para doentes mentais crónicos. Fonte: WHO Mental Health Atlas 2005.

\begin{tabular}{|l|c|c|c|}
\hline \multicolumn{1}{|c|}{$\begin{array}{c}\text { Níveis } \\
\text { Económicos dos } \\
\text { países baseados } \\
\text { na GDP (2004) }\end{array}$} & $\begin{array}{c}\text { Existência (\%) } \\
\text { de cuidados } \\
\text { integrados de } \\
\text { Psiquiatria na } \\
\text { comunidade }\end{array}$ & $\begin{array}{c}\text { Existência (\%) } \\
\text { de Políticas de } \\
\text { Beneficência para } \\
\text { os desfavorecidos }\end{array}$ & $\begin{array}{c}\text { Existência (\%) } \\
\text { de serviços } \\
\text { especializados para } \\
\text { dentes mentais } \\
\text { crónicos }\end{array}$ \\
\hline 1 Inferior & 51.7 & 55.2 & 55.2 \\
\hline 2 Médio-Inferior & 51.9 & 88.7 & 44.4 \\
\hline 3 Médio-Superior & 90.9 & 78.8 & 72.7 \\
\hline 4 Superior & 97.4 & 100.0 & 86.8 \\
\hline
\end{tabular}


De acordo com o Atlas de Saúde Mental da OMS, podemos observar diferenças muito acentuadas na existência de serviços de saúde mental integrados na comunidade e de serviços especializados para doentes mentais crónicos (serviços de reabilitação psicossocial) (WHO, 2000, 2001 a, b, 2005).

Dos países de menor desenvolvimento económico, apenas metade fornece serviços psiquiátricos baseados na comunidade, benefícios por incapacidade e reabilitação, ao passo que dos países desenvolvidos e de nível mais elevado $97,4 \%$ e $100 \%$ dispõem de serviços descritos anteriormente e proporcionam benefícios.

Tabela 2. Distribuição do custo médio para tratamentos de manutenção de transtornos psicóticos com Chrorpromazine $400 \mathrm{mgr}$, por paciente, em função do nível de desenvolvimento económico dos países. Fonte: WHO Mental Health Atlas 2005.

\begin{tabular}{|l|c|}
\hline Nível Económico dos países baseado no GPD (2004) & Custo Médio em USDollars \\
\hline 1 Inferior & 47.89 \\
\hline 2 Médio-Inferior & 35.84 \\
\hline 3 Médio-Superior & 108.62 \\
\hline 4 Superior & 155.200 \\
\hline
\end{tabular}

Na tabela 2, verifica-se que o custo médio de manutenção do tratamento de transtornos psiquiátricos com 400 mgr de Chrorpromazine está positivamente relacionado com o nível económico dos países.

Finalmente, os dados da tabela 3 mostram que o nível económico dos países está definitivamente relacionado com o desenvolvimento dos serviços especializados (reabilitação, etc.) para crianças e adultos. Nos países de nível económico inferior, apenas 34,5\% e 17,9\% presta esses serviços para crianças e idosos, respectivamente, ao contrário dos 86,8\% e $89,5 \%$ dos países ricos, que oferecem estes serviços, respectivamente, a crianças e adultos. 
Tabela 3. Programas disponíveis especializados de saúde mental para crianças e idosos em função do nível de desenvolvimento económico dos países. Fonte: WHO Mental Health Atlas 2005.

\begin{tabular}{|l|c|c|}
\hline $\begin{array}{c}\text { Níveis Económicos dos países } \\
\text { baseados na GPD (2004) }\end{array}$ & $\begin{array}{c}\text { Para Crianças } \\
\text { Países (\%) }\end{array}$ & $\begin{array}{c}\text { Para Idosos } \\
\text { Países (\%) }\end{array}$ \\
\hline 1 Inferior & 34.5 & 17.9 \\
\hline 2 Médio-Inferior & 73.6 & 50.9 \\
\hline 3 Médio-Superior & 72.7 & 66.7 \\
\hline 4 Superior & 86.8 & 89.5 \\
\hline
\end{tabular}

Existem outros resultados importantes, como a percentagem do orçamento total da saúde dedicada à saúde mental que variou entre 0,5\% a 1,2\% nos países de nível económico baixo, foi de 2,8\% em países de nível económico médio e alcançou 6,9\% nos países de nível económico alto. A escassez de profissionais de saúde mental (psiquiatras, psicólogos, assistentes sociais, enfermeiros psiquiátricos, etc) é profunda entre os países de economia baixa (Saraceno \& Barbui, 1997).

No entanto, em muitos dos países de fracos rendimentos, as famílias e redes comunitárias de apoio ainda conseguem existir procurando dar apoio e aliviar o sofrimento dos seus membros com doenças mentais (Jablensky et al., 1992; Mc Kenzie et al., 2004).

O outro lado da relação entre economia e doença mental na era da globalização tem a ver com os aspectos sociais ligados ao movimento da desinstitucionalização psiquiátrica em países desenvolvidos (Williams et al., 1973; Scull, 1985; Thornicroft \& Bebbington, 1989).

Ainda assim, o processo contínuo da desinstitucionalização em pacientes institucionalizados por um longo período de tempo em países ocidentais está a envolver cada vez mais a família e a comunidade. Quando a família e a comunidade local não têm possibilidades económicas não podem receber os pacientes desinstitucionalizados. Assim, é muito provável que estes indivíduos se tornem pessoas "sem-abrigo" ou trans- institucionalizados. A trans-institucionalização (passagem das pessoas por diversas 
instituições) é um fenómeno novo em que um grande número de doentes mentais crónicos se encontra em prisão por delitos cometidos. Nestes casos, a reabilitação psicossocial soa a ironia.

\section{A política económica da desinstitucionalização psiquiátrica}

A desinstitucionalização, para além da negação teórica dos asilos, incorpora o factor custo-benefício ao colocar na comunidade doentes crónicos com anos de internamento, dado o facto de a maioria desses doentes pertencerem a classes sociais médias e baixas (Bachrach, 1976). Nos meados dos anos 70, quando a N.I.M.H. nos EUA iniciou o programa nacional de fechar os Hospitais Psiquiátricos Estaduais, a primeira Comissão de Saúde Mental criada pelo presidente Jimmy Carter focou-se no desenvolvimento de programas especiais para pacientes que saíam para a comunidade após longos anos de internamento. Contudo, quando o presidente Ronald Reagan tomou o poder em 1981, a política relativa à saúde mental deixou de ser uma prioridade federal, teve sérios cortes orçamentais, assim como o bloqueio de uma série de regalias. Neste período, nos EUA, a "Medicaid", a "Supplemental Security Income" e a "Section 8 housing" cobriram de forma muito precária as necessidades dos doentes mentais crónicos. Nesse mesmo período, os utentes dos hospitais e dos serviços na comunidade organizaram-se no quadro da "National Alliance for the Mentally Ill" (Aliança Nacional dos Doentes Mentais) para defenderem os seus direitos (Mechanic, 2008).

Nos anos 90, foi iniciada nos EUA, uma tentativa para controlar as despesas com os vários cuidados e tratamentos, que ficou conhecida por "Managed Behavioral Health Care (MBHC) " tanto para o sector público como para o sector privado. Foi a partir de então que começaram a ser evitadas as hospitalizações e se procurou reduzir o tempo dos internamentos. Este sistema (MBHC) apresentou sérias falhas nos cuidados a prestar às pessoas com doença mental prolongada pois estes casos requerem serviços especializados, com alta intensidade e que não são disponibilizados por este sistema (Mechanic, 2008). 
A desinstitucionalização é um processo multifuncional que requer uma via de avaliação das necessidades socioeconómicas dos doentes que vão ser "devolvidos" à comunidade e o desenvolvimento de um sistema alternativo de cuidados (Mechanic, 1990; Madianos, 2002).

Por outro lado, a esquizofrenia é, em muitos casos, a doença de que sofre a maioria dos doentes desinstitucionalizados, e constitui uma doença demasiado debilitante que acarreta sérios problemas económicos. Nos EUA foi estimado que o custo total inerente à esquizofrenia em 2002 foi de $\$ 62.7$ biliões de dólares, sendo $\$ 22.7$ biliões referentes a cuidados de saúde directos, $\$ 7.0$ biliões referentes a doentes com alta clínica, $\$ 5.0$ biliões para medicamentos, $\$ 2.8$ biliões referentes ao tratamento de doentes internados e $\$ 8.0$ biliões referentes a cuidados de longo prazo (Wu et a.l, 2005). O número total de custos directos não referentes à saúde foi estimado em $\$ 7.6$ biliões e o número total de custos indirectos foi estimado em $\$ 32.4$ biliões.

Da mesma forma, no vizinho Canadá, os custos directos com os cuidados de saúde e os custos não atribuídos a cuidados de saúde, tendo em consideração o número estimado de pessoas com esquizofrenia ( $\mathrm{n}=$ 234,305), foram estimados em 2,02 bilhões de dólares CAN, no ano de 2004. Isto combinado com a elevada taxa de desemprego deste país, com a perda de produtividade e com um aumento da taxa de mortalidade e morbidade, resultou numa estimativa do custo adicional de 4,83 biliões de dólares CAN (Morrow, 2007).

Na Austrália, a estimativa do custo anual para as psicoses é de cerca de 2,25 biliões de dólares australianos (Neil et al., 2003).

Os valores económicos acima referidos mostram que a esquizofrenia afecta cem mil pessoas, cuja maioria foi desinstitucionalizada, com uma elevada carga económica que exige uma atribuição de recursos, não uma simples política de redução de camas de hospital e que as abandona ao seu destino na comunidade. Alguns estudos têm mostrado uma correlação negativa entre a redução das camas hospitalares e as taxas de mortalidade excessiva (Saha et al., 2007). A negligência de cuidados médicos a tais doentes é refletida na sua maior mortalidade e morbidade. Pessoas com 
doença mental crónica morrem, em média, 20-25 anos mais cedo do que na população geral (Parks et al., 2006).

Vários estudos documentam que há um grave problema de saúde pública para as pessoas sub-atendidas por sistemas de saúde mental (Yanos et al., 2001). A maioria (60\%) das mortes prematuras destes doentes é devida a condições médicas (que geralmente podem ser prevenidas), tais como doenças cardiovasculares, doenças pulmonares e infecções. Outras 30-40\% são causadas pelo suicídio, por lesões e acidentes. Entre estas pessoas, as causas naturais de morte são as doenças cardiovasculares, complicações por diabetes e síndrome metabólica, doenças respiratórias causadas pelo tabaco, e infecções incluindo o HIV/SIDA e a tuberculose (esta foi "encontrada" recentemente entre os residentes em lares e centros de sem-abrigo). Graves problemas de saúde estão associadas à desnutrição, obesidade, falta de exercício, excesso de fumo, consumo de álcool e comportamento sexual inseguro. O risco relativo de doenças cardiovasculares é 2 vezes superior em pessoas que sofrem de esquizofrenia devido a obesidade excessiva, 2-3 vezes por fumar, duas vezes por diabetes e 5 vezes maior para dislipidemia. Sabe-se que $18-20 \%$ destas pessoas sofrem de hipertensão. É uma verificação comum que as pessoas com doença mental grave levam muitas vezes uma vida marginal nas áreas mais desfavorecidas dos centros urbanos, em condições extremas de baixa qualidade, com hábitos de vida degradantes, com consumos abusivos de substâncias ilícitas. Estas são muitas vezes vítimas de violência e roubo, com pouco acesso a todas as formas de cuidados de saúde devido à falta de seguro ou à sua falta de motivação, vivem com medo constante e instabilidade social (conflitos e estigma), devido à fragmentação do sistema de saúde mental (Wilton, 2004).

\section{Conclusão}

Torna-se evidente que, em muitos países ocidentais, os indivíduos portadores de doença mental crónica desinstitucionalizados são vulneráveis a uma série de problemas de saúde e de carácter social, enfren- 
tando grandes dificuldades no acesso aos serviços de saúde. Nos E.U.A., as pessoas com doença mental grave, devido à condição da sua classe social, são encaminhados para os sistemas de cuidados de saúde mental sub-financiados pelos orçamentos públicos. Tais serviços públicos de saúde mental procuram superar uma série de obstáculos, como a falta de reembolso pelas acções de educação para a saúde e apoio familiar, falta de serviços e de pessoal para acompanhamento e adequada gestão de casos (case management service), má coordenação entre os serviços e falta de tratamento integrado nos casos em que há co-ocorrência de distúrbios psiquiátricos, abuso de substâncias ilícitas e deficientes condições de saúde geral, a exigir vigilância médica.

Por último, registe-se que o fenómeno da trans-institutionalização complementa a política de desinstitucionalização. Nos E.U.A., há cerca de 200.000 pessoas apreendidas com doença mental grave nas prisões do Estado ou federais. Pode-se concluir que a desinstitucionalização foi muitas vezes associada com atitudes e reacções negativas da comunidade, como preconceitos, estereótipos, estigma e discriminação contra a reintegração na comunidade de pessoas com doenças mentais graves (Matschinger \& Angermeyer, 2004). No entanto, o estigma e atitudes negativas podem ser alterados se forem feitos esforços adequados para intervenções sistemáticas na comunidade sobre saúde mental (Madianos et al., 1987 \& Madianos et al, 1999).

Em suma, as motivações de carácter ideológico, económico e de política social que estão por trás do movimento de desinstitucionalização nos países ocidentais são: Ideológicas, o caso da reforma italiana (Warner, 1994); Económicas, redução de custos e do valor dos edifícios de asilo, o caso de E.U.A. e Grã-Bretanha; e Humanitárias, preocupações para a libertação dos internados, o caso da França, Alemanha, Espanha, Grécia e outros países europeus (Madianos, 1994).

Em Itália, particularmente, a desinstitucionalização tornou-se sinónimo de "Psychiatria Democratica", concepção ideológica com ecos do movimento de Maio de 1968, que influenciou o encerramento de todos os hospitais psiquiátricos públicos. Na Grécia, Espanha e alguns outros 
estados europeus, a Reforma Psiquiátrica foi a primeira prioridade, que precedeu à abertura dos grandes hospitais psiquiátricos (Madianos, 1974, Madianos \& Christodoulou, 2007). Nos E.U.A, a desinstitucionalização foi realizada em circunstâncias sem alternativas adequadas, abandonando os internados "à sua sorte", tendo como resultado os fenómenos de porta giratória e o aumento do número de pessoas sem-abrigo. Existem hoje, em todas as principais cidades dos E.U.A., cerca de um milhão de pessoas desabrigadas portadoras de doenças mentais crónicas.

A desinstitucionalização provou ser bem-sucedida nos países em que a reforma psiquiátrica passou a ser a prioridade e foi completada com um sistema abrangente de alternativas baseadas na comunidade e nos serviços de segurança social e bem-estar, designadamente na Grã-Bretanha e nos países escandinavos, em que a Psiquiatria comunitária mais se desenvolveu. Isso significa que o processo de desinstitucionalização tem de ser conduzido passo a passo pois é multidimensional e deve ser sempre centrado nas necessidades das pessoas doentes. A desinstitucionalização engloba as necessidades das pessoas ao longo da vida, incluindo a continuidade de tratamentos (cuidados de saúde geral e de saúde mental), habitação, emprego, educação, sistema de apoio comunitário e serviços complementares (desporto, lazer, etc.). Se o apoio da família existe, o Estado alivia a carga dos cuidados a manter. O objectivo final é a concretização do direito da pessoa que sofre a uma posição autónoma e inserção na comunidade, num estatuto completo de recuperação social e clínica e de cidadania plena.

\section{Referências bibliográficas}

ASTRACHAN, B. M., BrAUER, L., HARROW, M., SCHWARTZ, C. C. (1974). Symptomatic outcome in schizophrenia. Archives General Psychiatry. 31, 155-159.

BACHRACH, L. L. (1976). Deinstitutionalization: An analytical review and sociological review. Rockville M.D. National Institute of Mental Health.

BRENNER, H. (1973). Mental Illness and the economy Cambridge Mass. Harvard University Press.

CURRIER, G. (2000). Datapoints: Psychiatric bed reductions and mortality among persons with mental disorders. Psychiatric Services, 51, 851-854. 
Drew, D., Drebing, C., VAn Ormer, A., Losardo, M., Krebs, C., Perk, W., Rosenheck, R. (2001). Effects of disability compensation on participation in and outcome of vocational rehabilitation. Psychiatric Services, 52, 1479-1484.

FARRIS, R. \& DUNHAM, H. (1939). Mental disorders in urban areas. Chicago: Chicago University Press.

HollingsheAd, A. B. \& REDliCH, F. (1958). Social class and mental illness. New York: J. Wiley.

JAblensky, A., SARTorius, N., ERnberg, G., et al. (1992). Schizophrenia: Manifestations, incidence and course in different cultures: A World Health Organization ten-country study. Psychological Medicine Supplement 20.

MADIANOS, M. (1994). Recent advances in community Psychiatry and Psychosocial Rehabilitation in Greece and the other southern European countries. International Journal of Social Psychiatry, 40, 157-164.

MADIANOS, M. (2002). Deinstitutionalization and the closure of public mental hospitals. International Journal of Mental Health, 31, 66-75.

MAdiAnos, M. (2006). Psychiatric Rehabilitation in the era of globalization. World Psychiatry, 5, 163-164.

MAdianos, M., CHRistodoulou, G. (2007). Reform of the mental healthcare system in Greece, 1984-2006. International Psychiatry, 2, 16-19.

MAdianos, M., ECONOMOU, M., DAFni, O. et al. (2004). Family disruption, economic hardship and psychological distress in schizophrenia: can they be measured? Eur Psychiatry., 19, 408-414.

madianos, M., Economou, M., hatjiandreou, M., PApageOrgioU, A., RogakoU, E. (1999). Changes in public attitudes towards mental illness in the Athens area (1979/1980-1994). Acta Psychiatrica Scand., 99, 73-78.

Madianos, M., Madianou, D., Vlchonikolis, J., STefanis, C. (1987). Attitudes towards Mental Illness in the Athens area: Implications for community mental health intervention. Acta Psychiatrica Scand., 75,158-165.

MATSCHinger, H., ANGERMEYER, M. (2004). The public's references concerning the allocation of financial resources to mental health care: results from a representative population survey in Germany. European Psychiatry, 19, 478-82.

MCKENZIE, K., PATEL, V. \& ARAYA, R. (2004). Learning from low income countries: mental health. British Medical Journal, 13, 1138-41.

MECHANiC, D. (2007). Mental health services, then and now. Health Affairs, 26, 1548-1550 .

MeChanic, D. (2008). Mental health and Social Policy: Beyond Managed Care. $5^{\text {th }}$ Ed. Boston: Allyn \& Bacon.

MECHANIC, D. \& ROCHEFORT, D. (1990). Deinstitutionalization: An appraisal of reform. Annual Review of Sociology, 16, 301-327.

MORA, G. (1975). Historical and the theoretical trends in Psychiatry. In H. Freedman, B. Kaplan \& B. Sadock (Eds). Comprebensive Textbook of Psychiatry Vo. Baltimore: Williams Wilkins.

MORROW, M. (2004). Mental health reform, economic globalization and the practice of citizenship. Can J Commun Ment Health, 23, 39-50.

Murray, C., LOPEZ, A. (1996). The global burden of disease. Geneva: World Health Organization, Harvard School of Public Health and the World Bank. 
MYERS, J. \& BEAN, L. (1968). A decade later: A follow up of social class and mental illness. New York, J. Wiley.

NEIL, A., LEWIN, T. \& CARR, V. (2003). Allocation of resources and psychosis. Australian and New Zealand Journal of Psychiatry, 37, 15-23.

ODEGARD, O. (1964). Pattern of discharge from Norwegian Psychiatric hospitals before and after the introduction of the psychotropic drugs. American Journal of Psychiatry, 120, 772-778.

PARKS, J., SVEDEN, D., SINGER, P. \& FOTI, M. H. (2006). Morbidity and Mortality in people with serious Mental Illness. Technical Report. Alexandria VA, National Association of State Mental Health Program Directors.

RALPH, D. S. (1983). Work and madness: The rise of community psychiatry. Montreal: Black Rose.

ROTHMAN, D. (1971). The discovery of asylum. Boston: Little Brown.

SAHA, S., ChANT, D. \& MCGRATH, J.. (2007). A systematic review of mortality in schizophrenia. Archives of General Psychiatry, 64, 1123-1131.

SARACENO, B. \& BARbui, C. (1997). Poverty and mental illness. Canadian Journal of Psychiatry, 42, 285-90.

SCHENe, A., ViJngaARden, VAN B. \& KoeTER, M. W. J. (1998). Family caregiving in schizophrenia: domains and distress. Schizophr Bull, 24, 609-618.

SCULL, A. (1985). Deinstitutionalization and public policy. Social Science and Medicine, 5 , 545-562.

Thornicroft, G. \& BebBington, P. (1989). Deinstitutionalization-From hospital closure to service development. British Journal of Psychiatry, 155, 739-753.

WARNER, R. (1994). Recovery from schizophrenia: Psychiatry and political economy. New York: Routledge.

WAVE, N. \& GOLDFInGERS, S. (1997). Poverty and rehabilitation in severe psychiatric disorders. Psychiatr Rehab J., 21, 3.

Williams, G., HSU, F. T. \& LIN, T. Y. (1973). Prediction of the burden of released mental patients. Community Mental Health, 9, 303-15.

Wilton, R. (2004). Putting policy into practice? Poverty and people with serious mental illness. Soc Sci Med, 58, 25-39.

WING, J. K. \& BROWN, G. W. (1970). Institutionalism and Schizophrenia. London: Cambridge University Press.

WORLD HEALTH ORgANiZATION. (2000). The World Health Report 2000 - Health systems: improving performance. Geneva: World Health Organization.

WORLD HEALTH ORgANiZATION (2001a). The World Health Report 2001 - Mental health: new understanding, new hope. Geneva: World Health Organization.

WORLD HEALTH ORGANIZATION (2001b). Atlas: Mental health resources in the world 2001. Geneva: World Health Organization.

WORLD HEALTH ORGANIZATION (2005). Atlas: Mental health resources in the world. Geneva: World Health Organization.

Wu, E. Q., Birnbaum, H. G, Shil, L., Ball, D. E., Kessler, R. C., Moulis, M. \& AGGarwal, J. (2005). The economic burden of schizophrenia in the Unites States in 2002. Journal of Clinical Psychiatry, 66, 1122-1129.

YANOS, P., ROSENFIELD, S. \& HOWRWITZ, A. (2001). Negative and supportive social interactions and quality of life among persons diagnosed with severe mental illness. Commun Ment Health J., 37, 405-419. 